\title{
Sequential versus concomitant chemoradiotherapy in locally advanced head and neck cancer
}

\author{
Hesham Tawfik and Lamiss Mohamed Abd El-Aziz \\ Clinical Oncology Department, Faculty of Medicine, Tanta University Hospital
}

\begin{abstract}
Purpose: To test the feasibility of neoadjuvant chemotherapy followed by concomitant chemoradiotherapy regimen versus concomitant chemoradiotherapy in locally advanced squamous cell carcinoma of the head and neck cancer.
\end{abstract}

Material and Methods: Patients with Stage III and IVA and B squamous cell carcinoma of head and neck excluding nasopharynx with the following criteria were enrolled ; PS 0-2; no prior surgery, chemotherapy or radiotherapy ( $\mathrm{CT}$ or RT) were prospectively randomly assigned to receive 3 cycles of neoadjuvant TPF(arm A: T ( docetaxel) $75 \mathrm{mg} / \mathrm{m} 2, \mathrm{~d} 1$; P (platinol) $75 \mathrm{mg} / \mathrm{m} 2, \mathrm{~d} 1 ; 5$-flurourcil $750 \mathrm{mg} / \mathrm{m}^{2} \mathrm{IV}$,D1-5) followed the concurrent chemoradiotherapy (CT/RT) ( two cycles of cisplatin $20 \mathrm{mg} / \mathrm{m}^{2}$, days $1-4$, plus 5 -fluorouracil $750 \mathrm{mg} / \mathrm{m}^{2} /$ day IV D1-4during weeks 1 and 6 of radiotherapy).

Concurrent chemoradiotherapy (arm B) consisted of two cycles of cisplatin $20 \mathrm{mg} / \mathrm{m}^{2}$, days $1-4$, plus 5-fluorouracil $750 \mathrm{mg} / \mathrm{m}^{2} /$ day IV D1-4 during weeks 1 and 6 of radiotherapy. Basic demographics and clinical characteristics", overall survival rate, locoregional or systemic relapse rates and time to relapse were recorded. Results: A total of 49 patients were enrolled in our study between march 2010 and march 2014, with (24 arm $\mathrm{A}$ and $25 \mathrm{arm} \mathrm{B}$, four not evaluable in both group. Following CT/RT, The complete response rate was $52.4 \%$ and $20 \%$ in arm A and B respectively which was statistically significant $(P=0.022)$.

Median follow-up of all patients was 38 months (range 7-42 months). Three year overall survival rates $72.7 \%$ in arm $\mathrm{A}$ and $68.7 \%$ in arm $\mathrm{B}$, respectively which was statistically was insignificant $(P$ value $=0.4113)$. Three year distant disease-free survival rate in group A was $65.8 \%$ versus $60 \%$ in group B which was statistically insignificant $(P$ value $=0.3772)$. The three year progression - free survival was higher in group A than group B $61.1 \%$ versus $53.8 \%$ which was statistically insignificant $(P$ value $=0.3345)$.

The primary endpoint was complete response evaluated 6 weeks after the end of chemoradiotherapy While Secondary endpoints included time to disease progression (TTP), survival and safety.

Conclusion: Induction TPF followed by CT/RT was associated with higher CR in patients with locally advanced SCCHN without unaccepted interruption of the treatment.

Key words: head and neck squamous cell carcinoma, chemoradiotherapy

Corresponding Author: Lamiss Mohamed Abd El-Aziz

E-mail: lamissmohamed@yahoo.com

\section{INTRODUCTION}

Head and neck squamous cell carcinomas (HNSCC) are the sixth most common cancers ${ }^{1}$, with around two thirds of patients presenting with locally advanced disease ${ }^{2}$.

The treatment of advanced disease poses a major challenge in terms of balancing tumor outcomes with acceptable toxicity and maintaining organ function ${ }^{3,4}$. For many years primary surgery and/or radiotherapy have been the mainstay of treatment ${ }^{5}$.

Organ preservation using concomitant chemoradiotherapy has been accepted as an alternative to surgery ${ }^{6-7}$.
The role of chemotherapy has gradually emerged, and is now taking a more prominent place in treatment algorithms for locally advanced HNSCC. The use of concurrent chemoradiotherapy has improved locoregional control, with optimal results being achieved with cisplatin $^{6,7}$. Induction chemotherapy has been used in an attempt to gain the benefit of full therapeutic doses of chemotherapy via additive clonogen cell kill and spatial cooperation to treat distant micro metastatic disease, whilst avoiding the enhanced toxicity of concurrent treatment $^{8-9}$.

Here we present the outcomes for patients with locally advanced stage III-IVA \&B HNSCC managed with 
Kasr-El-Aini Journal Of Clinical Oncology And Nuclear Medicine

Vol. 10 | No. 1-2 $2014 \quad$ Hesham Tawfik and Lamiss Mohamed

induction chemotherapy followed by chemoradiotherapy versus chemoradiotherapy alone.

\section{PATIENTS AND METHODS}

From March 2010 to March 2014 forty nine patients with histologically documented locally advanced stage III- IVA\& $\mathrm{B}^{10}$ head and neck squamous cell carcinoma were prospectively randomized (1:1 ratio) this study at Clinical Oncology Department, Faculty of Medicine, Tanta University Hospital. Each patient was required to meet the following criteria: age between 18 and 65 years; Eastern cooperative oncology group (ECOG) status0-2; no previous surgery, chemotherapy or radiotherapy treatment; measurable disease; adequate hematological, hepatic, and renal functions.

Institutional scientific and ethical committees approval was obtained before the start of trial, Patients with severe peripheral neuropathy, symptomatic heart failure, sever arrhythmia, active infection, pregnant or lactating mothers, active peptic ulcer disease, clinical hearing loss, and uncontrolled diabetes mellitus, any other uncontrolled medical illness, were considered as a contraindication for inclusion.

Group A (25patients) was treated with three cycles of chemotherapy followed by concurrent chemoradiotherapy. Group B (24 patients) were treated with concurrent chemoradiotherapy.

Four patients in group A were non evaluable for response, 2 due to protocol violation and 2 for irregular treatment schedule. In group B 4 patients were excluded, one dropped out, 1 due to protocol violation and 2 due to irregular treatment schedule. Evaluable patients for response were 21 in group A and 20 in group B Figure (1)

\section{Treatment Plan \\ Induction chemotherapy}

Induction chemotherapy consisted of 3 cycles of TPF; (Docetaxel) $75 \mathrm{mg} / \mathrm{m}^{2}$ IV over half an hour, D1; P (Platinol) $75 \mathrm{mg} / \mathrm{m}^{2}$ IV over one hour, D1; 5-flurourcil $750 \mathrm{mg} / \mathrm{m}^{2}$ IV over 6 hours, D1-5 every three weeks. Hydration and adequate anti-emetic therapy were ensured for all patients (5HT3 antagonists and dexamethasone). Prophylactic granulocyte colony stimulating factor (G-CSF) was not allowed, but G-CSF was given to patients who experienced grade III neutropenia and febrile neutropenia.

\section{Radiotherapy}

Thermoplastic casts with two point stabilization of the head were used to immobilize all patients in suitable anatomic positions. Computed Tomography
(CT) simulation was marked on the individual patient. Slice thickness was $3-5 \mathrm{~mm}$. The mask of the patient was marked with radioopac labels with the help of laser beams.

Virtual simulation: The 3D conformal treatment plan was performed in consistency with ICRU (International Committee of Radiation Units and measurements) 50 and ICRU 62 guidelines ${ }^{11-13}$. The findings on clinical examination and CT and/ or MRI before RT were used to constitute the GTV (Gross Tumor Volume), the CTV (Clinical Target Volume) and the PTV (Planning Target Volume). GTV tumor delineation was done to include the primary tumor and GTV node consisted gross lymphatic metastasis. CTV (tumor and node) volumes were constructed by adding margins to GTV volumes as to clinical protocols and experiences for probable microscopic extension of disease. PTV volumes were planned by adding $0.5 \mathrm{~cm}$ to the $\mathrm{CTV}$, for possible set-up errors. Internal margin has been neglected in this study

Radiation therapy was given using 3D conformal radiation technique. After casting the customized thermoplastic mold, computerized tomography scan simulation was done. The images were then transferred to planning system. After drawing the target volumes and organs at risk on the planning scans, external beam radiation was delivered to the primary and the nodal areas using $6 \mathrm{MV}$ Linear Accelerator photons. A total dose of 6600-7000 cGy/33-35 fractions ; 200cGy/ fraction five days a week over a period of six to seven weeks was delivered.

The definitive curative radiation dose administered to the primary tumor was between 70 and 74 Gy, administered as fractions of 2 Gy per day 5 days per week. The dose administered to uninvolved lymph nodes was at least $50 \mathrm{~Gy}$. Involved lymph nodes were to receive 60 to $74 \mathrm{~Gy}$, depending on whether an elective neck dissection was indicated after completion of treatment or not.

\section{Concomitant chemotherapy}

Two cycles of cisplatin $20 \mathrm{mg} / \mathrm{m}^{2}$ were given intravenous infusion(one hour), days1-4, plus 5-fluorouracil $750 \mathrm{mg} / \mathrm{m}^{2} /$ day intravenous infusion (4-6 hours), D1-4 during weeks 1 and 6 of radiotherapy.

\section{Primary and secondary endpoints}

The primary endpoint was complete response evaluated 6 weeks after the end of chemoradiotherapy While Secondary endpoints included time to disease progression (TTP), survival and safety.

\section{Patients' evaluation}

The pretreatment evaluation was conducted before the start of treatment. It consisted of history, physical 
examination, dental, dietary, speech assessment, complete blood cell count, routine chemistry measurements, electrolytes, chest $\mathrm{x}$ ray, and abdomenoplevis ultrasound. All patients were investigated and staged with endoscopy, biopsy, computed tomographic (CT) scanning and/or magnetic resonance imaging (MRI) of head and neck region.

Laboratory investigations were repeated before every induction chemotherapy, and weekly during concurrent chemoradiation treatment. Radiological imaging procedures were repeated four weeks after completion of induction chemotherapy and 6 weeks after concurrent chemoradiation treatment.

Following completion of induction and concurrent treatment phases, tumor response was routinely evaluated by a detailed clinical examination of the head and neck, endoscopy and CT or MRI imaging of the primary site and the neck. Patients with less than a complete response were evaluated for surgery. Patients who were considered candidate for surgery by the multi-disciplinary team underwent salvage surgery of primary site and/or neck dissection.

Subsequently, patients were followed up by Chest $\mathrm{X}$-ray, and abdominal ultrasonography every 3 months, CT scan and MRI of the primary site, endoscopy and primary site biopsy were done every 6 months.

\section{Response and toxicity assessment:}

Tumor was assessed according to WHO criteria ${ }^{14}$. A complete response (CR) was defined as the disappearance of all measurable lesion for $\geq 4$ weeks proved by histopathology. A partial response (PR) was defined as a decrease of $\geq 50 \%$ of the sum of the products of the greatest perpendicular lesion diameters for $\geq 4$ weeks with no evidence of new lesions. No change ( $\mathrm{NC}$ ) was defined as a $<50 \%$ decrease or $<25 \%$ increase in the product of the greatest perpendicular lesion diameters with no evidence of new lesions for $\geq 4$ weeks. Progressive disease (PD) was defined as an increase in any measurable lesions by $\geq 25 \%$ or the detection of new lesions. Patients with less than a complete response were evaluated for surgery. Patients who were considered suitable for surgery by the multi-disciplinary team underwent salvage surgery of primary site and/or neck dissection.

Toxicity was routinely documented prospectively using the NCIC-version 3.0 grading system for chemotherapy toxicity ${ }^{15}$, and the RTOG system for radiotherapy toxicity ${ }^{16-17}$.

\section{Statistical analysis}

The following endpoints were used for assessment: induction chemotherapy response, overall treatment response, progression-free survival (PFS), disease-free survival (DFS), and overall survival (OS). Survivals were analyzed using Kaplan-Meier curves ${ }^{18}$.overall survival (OS) was determined as the time between histological diagnosis and death. Time to local relapse and systemic relapse were determined as time between histological diagnosis and local/systemic relapse, respectively. Distant metastases free survival (DMFS) was defined between the time between histological diagnosis after primary treatment ends that the patient survived without distant metastases. Progression free survival was the time during and after treatment during which cancer treated and doesn't get worse.

Variables compared between patients who received sequential chemoradiotherapy or concurrent chemoradiotherapy using the test or the Fisher exact test, and the log-rank test was used to compare survival curves. A value of $<0.05$ was regarded as statistically significant in 2-sided tests. Kaplan-Meier methods were used to evaluate time to disease recurrence or death. Cox regression was used for univariate and multivariate analyses to determine the potential risk factors associated with disease-free survival and overall survival. All statistical analyses were performed using SPSS statistical software version 21 (SPSS, Chicago, IL, USA).

\section{RESULTS}

From March 2010 to March 2014, 49 patients with stage III and IVA\&B head and neck cancer were enrolled in the study. Median age was 53 years in both treatment groups. Group A (25patients) was treated with three cycles of chemotherapy followed by concurrent chemoradiotherapy. Group B (24 patients) were treated with concurrent chemoradiotherapy.

Four patients in group A were non evaluable for response, 2 due to protocol violation and 2 for irregular treatment schedule. In group B 4 patients were excluded, one dropped out, 1 due to protocol violation and 2 due to irregular treatment schedule. Evaluable patients for response were 21 in group A and 20 in group B Figure (1)

In group $\mathrm{A}$, twenty patients were males $(83.3 \%)$, while in group B eighteen patients (72\%) were male.

The main characteristics' of both groups of both groups are shown in table (1) a statistically significant differences between both groups

\section{Treatment Response}

After induction chemotherapy, complete response occurred in 8 patients (38.1), partial response occurred in seven patients $(33.3 \%)$ with an overall response of $71.4 \%$. 


Vol. 10 | No. 1-2 $2014 \quad$ Hesham Tawfik and Lamiss Mohamed

At the end of treatment the overall response was $81 \%$ $(17 / 21)$ and $80 \%(16 / 20)$ in group A \& B respectively which was statistically insignificant $(P=0.064)$. In group A $(\mathrm{TPF}+\mathrm{CRT}), 11 / 21$ patients $(52.4 \%)$ achieved complete response $(\mathrm{CR})$ was higher than in group $\mathrm{B}$ (CRT) 20\% (4/20) which was statistically significant $(P$ value $=0.0045$ ) Table (2).

In group A, 9 (44.6\%) underwent surgery after induction chemotherapy. Five patients with neck residual disease and 2 patients with residual disease both on the neck and primary site and two underwent neck dissection as initially N2-N3.

In group B, surgery was carried out in 13 of the 20 assessable patients $(65 \%)$. three patients with neck residual disease and 2 patients with residual disease both on the neck and primary site and 8 because they had initial stage N2-N3 disease.

\section{Survival outcome:}

Median follow-up of all patients was 38 months (range 7-42 months). Three year overall survival rates were $72.7 \%$ and $68.7 \%$ in arm $\mathrm{A}$ and $\mathrm{B}$, respectively which was statistically insignificant $(P$ value $=0.4113)$. Three year disease distant free survival rate in group A was $65.8 \%$ versus $60 \%$ in group B which was statistically insignificant $(P$ value $=0.3772)$. The three year progression -free survival was B $61.1 \%$ versus $53.8 \%$ in group A and B respectively which was statistically insignificant $(P$ value $=0.3345$ ) Figure (2) Table (3).

Time to disease progression was $21.5 \& 12$ in group A \&B respectively which was statistically significant (0.04).

\section{Prognostic factor}

In subgroup analysis, smoker and advanced disease 9 stage IV A \& B. Patients had significant lower three PFS and OAS (P value (0.05) Table (4)

In group $\mathrm{B}, \mathrm{OAS}$ was statistically higher in patients with hemoglobin level $>13$ than patients with hemoglobin level less or equal to $13 \mathrm{gm} / \mathrm{dL}(P$ value $=0.039)$, for stage III versus stage IV ( $P$ value $=0.034)$, for stage III $(P=0.033)$ and T2 versus T3 and T4 $(P$ value $=0.0007)$. Cancer larynx than oro or hypopharynx $(P=0.03)$.

We analyzed age, sex, performance status, cigarette smoking, hemoglobin level, treatment line, cancer stage, and primary tumor location as prognostic factors for survival in all patients. Univariate analysis revealed that site ( $P$ value $=0.023^{*}$, hazard ratio $[\mathrm{HR}]: 1.700,95 \%$ confidence interval [CI]: 1.077-2.683) had a significantly poor 3-year overall survival rate. Multivariate analysis revealed that site ( $P$ value $=0.001 *$, hazard ratio $[\mathrm{HR}]$ :
3.862, 95\% confidence interval [CI]: 1.709-8.726) and $\mathrm{T}$ stage $\left(P\right.$ value $=0.016^{*}$. HR $=3.033,95 \%$ CI $(1.235$ 7.449).

In univariate analysis, none of these factors were associated with distant metastases free survival (DMFS), while on multivariate analysis, site is only prognostic factor of poor DMFS ( $P$ value $=0.027 *, \mathrm{HR}=2.474,95 \%$ CI(1.106-5.533).

As regard progression free survival, both univariate and multivariate analysis revealed that site $(P \quad$ value $=0.002 *, \quad \mathrm{HR}=2.156,95 \% \mathrm{CI}(1.331-3.491)$ $;(P$ value $=0.003 *, \mathrm{HR}=3.769,95 \% \mathrm{CI}(1.555-9.271)$ respectively.

\section{Acute Toxicity \\ Induction chemotherapy}

In group $\mathrm{A}$, Grade 3 neutropenia occurred in 4 patients $(26.7 \%)$. Two patients $(13.3 \%)$ experienced grade 3 mucositis.

\section{Chemoradiotherapy}

During concurrent chemoradiotherapy, the incidence of hematologic and non-hematologic toxicities was more in the TPF plus chemoradiotherapy arm than in the chemoradiotherapy alone arm. The most common grade 3 hematologic toxicity was leucopenia in both groups (Table 4). The most frequent grade 3 nonhematologic toxicities were stomatitis and dysphagia (Table 4).No grade 4 hematologic or nonhematologic toxicity was reported in either arm.

\section{Late Toxicity}

Among15 surviving patients in group A, 2 (13.3\%) suffered from late ( $>6$ months) grade 3 dysphagia. Among 10 surviving patients in group B, one patient had grade 3 dysphagia and 2 had trismus.

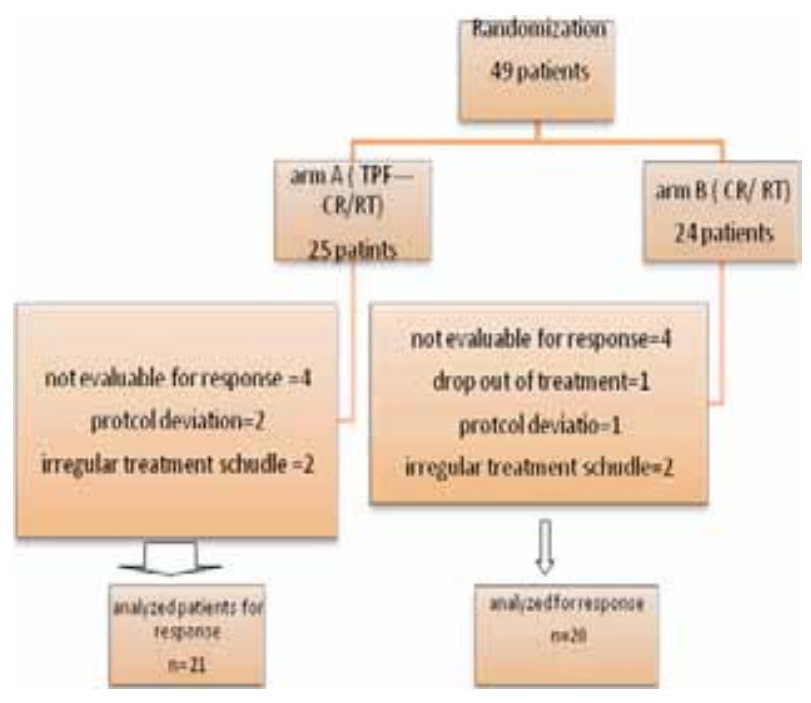

Figure 1: show patient flow chart 


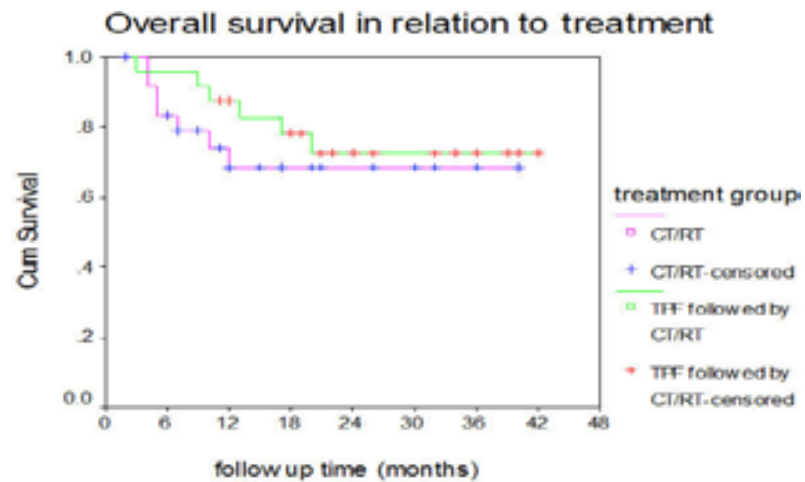

\section{Progression free survival in relation} treatment group

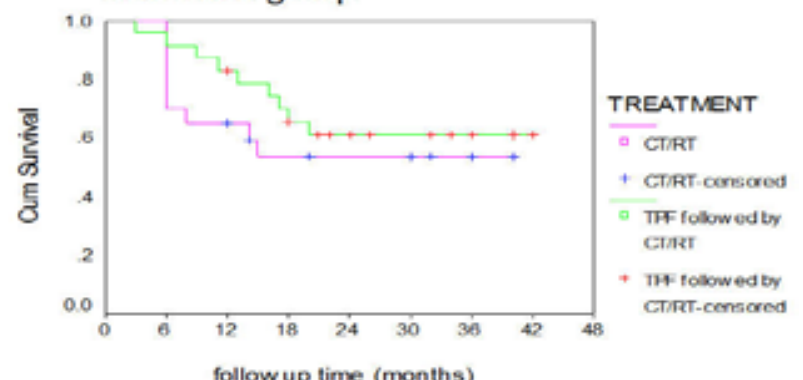

Distant metastasis free survival in relation to

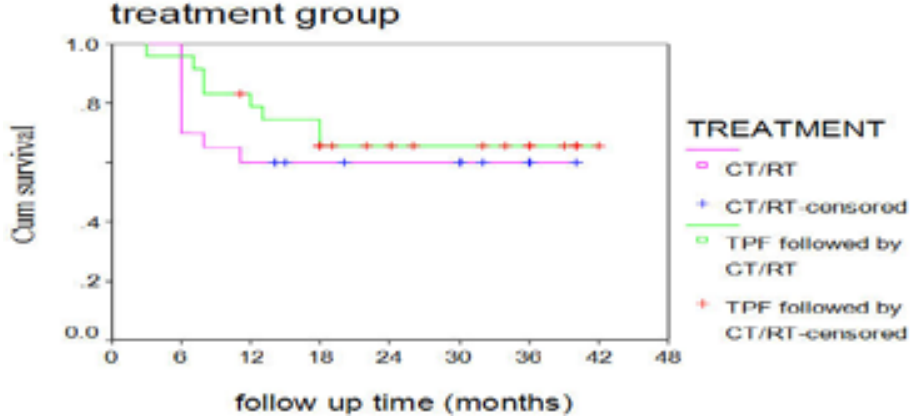

Figure 2: showed the overall, progression-free and distant metastasis free survival rates in relation to treatment group

Table 1: Main characteristics of all patients with locally advanced head and neck cancer in both study groups

\begin{tabular}{|c|c|c|c|c|c|}
\hline \multirow{3}{*}{ Patient characteristics } & \multicolumn{5}{|c|}{ Treatment group } \\
\hline & \multicolumn{2}{|c|}{ TPF+CRT(24) } & \multicolumn{2}{|c|}{ CRT(25) } & \\
\hline & No. & $\%$ & No. & $\%$ & \\
\hline Age (Range) & \multicolumn{2}{|c|}{$37-65 y$} & \multicolumn{2}{|c|}{$40-67$} & \\
\hline Median & \multicolumn{2}{|c|}{53} & \multicolumn{2}{|c|}{53} & \\
\hline \\
\hline Male & 20 & 83.3 & 18 & 72 & \\
\hline - $\quad$ Female & 4 & 16.6 & 7 & 28 & 0.273 \\
\hline \multicolumn{6}{|l|}{ Cigarette Smoking } \\
\hline Smoking & 14 & 58.3 & 15 & 60 & \\
\hline - $\quad$ Non-smoking & 10 & 41.7 & 10 & 40 & 0.906 \\
\hline \multicolumn{6}{|l|}{ Performance status } \\
\hline - $\quad 0 / 1$ & 19 & 79.2 & 14 & 56 & \\
\hline - $\quad 2$ & 5 & 20.8 & 11 & 44 & 0.077 \\
\hline \multicolumn{6}{|l|}{ Pretreatment Hb-level } \\
\hline - $\quad \leq 13 \mathrm{~g} / \mathrm{dL}$ & 16 & 66.7 & 21 & 84 & \\
\hline - $\quad>13 \mathrm{~g} / \mathrm{dL}$ & 8 & 33.3 & 4 & 16 & 0.158 \\
\hline \multicolumn{6}{|l|}{ Tumor site } \\
\hline - Oropharynx & 4 & 16.7 & 4 & 16 & \multirow{3}{*}{0.877} \\
\hline - $\quad$ Larynx & 16 & 66.6 & 18 & 72 & \\
\hline - $\quad$ Hypopharynx & 4 & 16.7 & 3 & 12 & \\
\hline \multicolumn{6}{|l|}{ Clinical stage } \\
\hline • $\quad$ III & 5 & 20.8 & 18 & 72 & \\
\hline IVA\&B & 19 & 79.2 & 7 & 28 & 0.376 \\
\hline \multicolumn{6}{|l|}{ T stage } \\
\hline $\mathrm{T} 2$ & 2 & 8.3 & 7 & 28 & \\
\hline $\mathrm{T} 3$ & 10 & 41.7 & 9 & 36 & \\
\hline • & 12 & 50 & 9 & 36 & 0.705 \\
\hline \multicolumn{6}{|l|}{ N stage } \\
\hline No & 3 & 12.5 & 2 & 8 & \\
\hline $\mathrm{N} 1$ & 8 & 33.3 & 4 & 16 & \\
\hline N2 & 11 & 45.8 & 12 & 48 & 0.474 \\
\hline $\mathrm{N} 3$ & 2 & 8.4 & 7 & 28 & \\
\hline
\end{tabular}




Vol. 10 | No. 1-2 $2014 \quad$ Hesham Tawfik and Lamiss Mohamed

Table 2: Response after the end of treatment in both groups.

\begin{tabular}{|c|c|c|c|c|c|}
\hline \multirow{3}{*}{ Response } & \multicolumn{5}{|c|}{ Treatment group } \\
\hline & \multicolumn{2}{|c|}{$\begin{array}{c}\text { TPF }+ \text { CRT (21) } \\
\text { Group A }\end{array}$} & \multicolumn{2}{|c|}{$\begin{array}{c}\text { CRT } \\
\text { Group B }\end{array}$} & \multirow[b]{2}{*}{$P$. value } \\
\hline & No. & $\%$ & No & $\%$ & \\
\hline CR & 11 & 52.4 & 4 & 20 & $0.022 *$ \\
\hline PR & 6 & 28.6 & 12 & 60 & $0.022 *$ \\
\hline $\mathrm{CR}+\mathrm{PR}$ & 17 & 81 & 16 & 80 & 0.064 \\
\hline SD & 3 & 14.3 & 1 & 5 & 0.157 \\
\hline DP & 1 & 4.8 & 3 & 15 & 0.157 \\
\hline Total & 21 & 100 & 20 & 100 & \\
\hline
\end{tabular}

Table 3: survival according to treatment group.

\begin{tabular}{lccc}
\hline Survival & Sequential chemoradiotherapy & Concurrent chemoradiotherapy & P value \\
\hline Three year Overall survival & $72.7 \%$ & $68.7 \%$ & 0.4113 \\
Three year PFS & $61.1 \%$ & $53.8 \%$ & 0.3345 \\
Three year DMFS & $65.8 \%$ & $60 \%$ & 0.3772 \\
\hline
\end{tabular}

Table 4: grade III hematological and non hematological toxicity in both study groups after end of treatment.

\begin{tabular}{lcc}
\hline & \multicolumn{2}{c}{ No of patients (\%) } \\
\cline { 2 - 3 } Acute toxicity & TPF+CRT & CRT \\
& $(\mathbf{2 1 )}$ & $\mathbf{( 2 0 )}$ \\
\hline Hematologic & & \\
Leucopenia & $5(23.8)$ & $3(15)$ \\
Neutropenia & $3(14.3)$ & $2(10)$ \\
Anemia & $3(14.3)$ & $2(10)$ \\
\hline Nonhematologic & & \\
Mucositis & $8(38.1)$ & $6(30)$ \\
Dysphagia & $5(23.8)$ & $3(15)$ \\
Skin reaction & $5(23.8)$ & $3(15)$ \\
Weight loss & $3(14.3)$ & $1(5)$ \\
\hline
\end{tabular}

\section{DISCUSSION}

Concurrent chemo-radiotherapy has been widely adopted as the standard of care for locally advanced HNSCC $^{19,20}$. Cisplatin is the chemotherapy agent of choice, with studies showing a 5-12\% improvement in long term survival with standard or altered fractionation regimens $^{21-22}$.

Induction chemotherapy followed by chemoradiotherapy is an alternative approach to concurrent treatment. It has shown a survival benefit in locally advanced HNSCC ${ }^{23-24}$.

Although it has only a minimal survival benefit of $2 \%$ in a large meta-analysis, the combination of cisplatin and 5 -FU was associated with a 5\% survival benefit ${ }^{25-28}$.

The role of systemic treatment in addition to radiotherapy in locally advanced HNSCC continues to develop. Concurrent chemo-radiotherapy remains a standard of care, while induction chemotherapy may have the same efficacy ${ }^{22}$. However, it remains uncertain whether combining induction with concurrent chemotherapy takes advantage of the benefits of both treatments. Studies are currently underway to investigate the potential superiority of induction chemotherapy followed by concurrent chemoradiotherapy compared with concurrent chemoradiotherapy alone.

This series of 49 patients reported here, demonstrates that induction chemotherapy can be successfully combined with concurrent chemoradiotherapy, without unaccepted toxicity. Radiotherapy commenced four weeks following the administration of the final cycle of chemotherapy. Therefore, induction chemotherapy did not preclude the prompt delivery of radiotherapy. Notably, by contrast with the EORTC/TAX323 trial ${ }^{26}$, patients in this series completed radiotherapy as planned. 
It should be noted that we used a similar chemotherapy protocol to that reported in ERTOC/TAX323 trial instead of five days) to minimize the expected grade 3 and but with $20 \%$ reduction in cisplatin and 5 fluorouracil dose and one day less ( 4 days instead of 5 days) to minimize the expected grade 3 and 4 toxicities that may interrupt radiotherapy ${ }^{27}$. Our protocol was similar to that reported by paccagnella et al. ${ }^{27}$.

Concomitant chemotherapy was given to all patients in our series; therefore it can be concluded that induction chemotherapy did not compromise patient fitness to commence definitive concurrent chemoradiation.

The overall toxicity of induction chemotherapy followed by (chemo)-radiotherapy appears acceptable. There were no on-treatment deaths.

In our series, the complete response rate was $52.4 \%$ $(11 / 21)$ and $20 \%(4 / 20)$ in arm A and B respectively which was statistically significant $(P=0.022)$.

Our results are nearly equal to that reported by Paccagnella et $a l^{28}$, in series of 101 patients were randomized to treatment: 50 patients received chemotherapy (three cycles of docetaxel, $75 \mathrm{mg} / \mathrm{m}^{2}$, and cisplatin, $80 \mathrm{mg} / \mathrm{m}^{2}$, on day 1 , plus $5-\mathrm{FU}, 800 \mathrm{mg} / \mathrm{m}^{2}$ as a 96-hour continuous infusion every 3 weeks) followed by the same chemoradiotherapy regimen in the other arm and 51 patients received chemoradiotherapy alone (two cycles of cisplatin, $20 \mathrm{mg} / \mathrm{m}^{2}$ on days $1-4$, plus 5 -FU, $800 \mathrm{mg} / \mathrm{m}^{2}$ as a 96-hour continuous infusion, on weeks 1 and 6 during radiotherapy, 66-70 Gy).The complete response was $50 \%$ and $21 \%$ in group $1 \& 2$ respectively.

However our results are lower than that that reported by Ghi et al. ${ }^{29}$, the CR rate was $62.5 \%$ for CRT and $80 \%$ for neoadjuvant TPF followed by CRT. This may be due the difference in the regimen given by Ghi et al. during the radiotherapy (carboplatin area under the curve 1.5 on Days $1-4$ and 5 -fluorouracil $600 \mathrm{mg} / \mathrm{m}^{2} / \mathrm{d}$ continuous infusion for $96 \mathrm{~h}$ ) starting on Days 1, 22, and 43 during RT).

Although complete response in the present series was higher in group A compare to group B, the difference in overall survival and disease distant free survival were statistically non significant which was similar to that reported by Paccagnella et al. ${ }^{28}$. The PARADIGM trial was a randomized phase III and reached the same conclusion with statistical significant advantage of neoadjuvant chemotherapy over concurrent chemoradiotherapy ${ }^{30}$. The DeCIDE study N2 and 3 disease reached the same conclusion $^{31}$.
In our series, time to disease progression was 21.5 \& 12months in group A and B respectively which was statistically significant $(P$ value $=0.04)$.

The analysis of different prognostic factors with survival (OAS, DMFS, PFS) revealed that the site was the significant prognostic factors on multivariate analysis $^{25,27}$. Also T stage was significant as regard oveall survival on multivariate analysis ${ }^{32}$.

The main limitation of the work was the small sample size and relatively short follow up.

\section{Conflict of interest}

No conflict of interest was declared.

\section{REFERENCES}

1. Siegel R, Naishadham D, Jemal A. Cancer statistics, 2012. CA Cancer.J.Clin. 2012 Jan-Feb;62(1):10-29.

2. Chandana SR, Conley BA. Neoadjuvant chemotherapy for locally advanced squamous cancers of the head and neck: current status and future prospects. Curr.Opin.Oncol. 2009 May;21(3):218-23.

3. Caudell JJS,P.E., Meredith RF, Locher JL, Nabell LM, Carroll WR, Magnuson JS, et al. Factors associated with long-term dysphagia after definitive radiotherapy for locally advanced head-and-neck cancer. Int.J.Radiat. Oncol.Biol.Phys. 2009;73(2):410-5.

4. Corry JD, Peters LJ, Rischin D. Optimising the therapeutic ratio in head and neck cancer. Lancet Oncol. 2010;11(3):287-91.

5. Ang KK. Multidisciplinary management of locally advanced SCCHN: optimizing treatment outcomes. Oncologist 2008;13(8):899-910.

6. Forastiere AA. Larynx preservation and survival trends: should there be concern? Head Neck 2010;32(1):14-7.

7. Olsen KD. Reexamining the treatment of advanced laryngeal cancer. Head Neck 2010;32(1):1-7.

8. Pignon JP, le Maître A, Maillard E, Bourhis J, MACHNC Collaborative Group. Meta-analysis of chemotherapy in head and neck cancer (MACH-NC): an update on 93 randomised trials and 17,346 patients. Radiother.Oncol. 2009;92(1):4-14.

9. Blanchard P, Baujat B, Holostenco V, Bourredjem A, Baey $\mathrm{C}$, Bourhis J, et al. Meta-analysis of chemotherapy in head and neck cancer (MACH-NC): a comprehensive analysis by tumour site. Radiother.Oncol. 2011;100(1):33-40.

10. Edge SB, Compton CC. The American Joint Committee on Cancer: the 7th edition of the AJCC cancer staging manual and the future of TNM. Ann.Surg.Oncol. 2010;17(6):1471-4.

11. ICRU News. ICRU Report 50, Prescribing, Recording, and Reporting Photon Beam Therapy. 1993.; Available: http:// www.icru.org/pubs.htm. 
12. Wambersie AandLandberg T. ICRU Report 62: Prescribing, Recording and Reporting Photon Beam Therapy (Supplement to ICRU Report 50. 1999; Available: http://www.icru.org/n_992_4.htm.

13. Chavaudra J, Bridier A. [Definition of volumes in external radiotherapy: ICRU reports 50 and 62].[Article in French]. Cancer Radiother. 2001;5(5):472-8.

14. Miller AB, Hoogstraten B, Staquet M, Winkler A. Reporting results of cancer treatment. Cancer 1981;47(1):207-14.

15. Trotti A, Colevas AD, Setser A, Rusch V, Jaques D, Budach $\mathrm{V}$, et al. CTCAE v3.0: development of a comprehensive grading system for the adverse effects of cancer treatment. Semin.Radiat.Oncol. 2003;13(3):176-81.

16. Cox JD, Stetz J, Pajak TF. Toxicity criteria of the Radiation Therapy Oncology Group (RTOG) and the European Organization for Research and Treatment of Cancer (EORTC). Int.J.Radiat.Oncol.Biol.Phys. 1995;31(5):1341-6.

17. Trotti A, Pajak TF, Gwede CK, Paulus R, Cooper J, Forastiere A, et al. TAME: development of a new method for summarising adverse events of cancer treatment by the Radiation Therapy Oncology Group. Lancet Oncol. 2007;8(7):613-24.

18. Kaplan EL, Meier P. Nonparametric Estimation from Incomplete Observations. Journal of the American Statistical Association 1958;53(282):457-81.

19. Prestwich RJ, Kancherla K, Oksuz DC, Williamson D, Dyker KE, Coyle C, et al. A single centre experience with sequential and concomitant chemoradiotherapy in locally advanced stage IV tonsillar cancer. Radiat.Oncol. 2010;5:121.

20. Goel AK, Singh D. Radiosensitization of head and neck cancer by targeting the epidermal growth factor receptor. Indian J.Med.Paediatr.Oncol. 2009;30(3):92-4.

21. Blanchard P, Baujat B, Holostenco V, Bourredjem A, Baey $\mathrm{C}$, Bourhis $\mathrm{J}$, et al. Meta-analysis of chemotherapy in head and neck cancer (MACH-NC): a comprehensive analysis by tumour site. Radiother.Oncol. 2011;100(1):33-40.

22. Krstevska V. Radiotherapy and chemotherapy in locally advanced head and neck squamous cell carcinoma. J.BUON. 2009;14(3):361-73.

23. Rapidis AD, Trichas M, Stavrinidis E, Roupakia A, Ioannidou $\mathrm{G}$, Kritselis $\mathrm{G}$, et al. Induction chemotherapy followed by concurrent chemoradiation in advanced squamous cell carcinoma of the head and neck: final results from a phase II study with docetaxel, cisplatin and 5-fluorouracil with a four-year follow-up. Oral Oncol. 2006;42(7):675-84.
24. Bhide SA, Ahmed M, Barbachano Y, Newbold K, Harrington KJ, Nutting CM. Sequential induction chemotherapy followed by radical chemo-radiation in the treatment of locoregionally advanced head-and-neck cancer. Br.J.Cancer 2008;99(1):57-62.

25. Posner MR, Hershock DM, Blajman CR, Mickiewicz E, Winquist E, Gorbounova V, et al. Cisplatin and fluorouracil alone or with docetaxel in head and neck cancer. N.Engl.J.Med. 2007;357(17):1705-15.

26. Vermorken JB, Remenar E, van Herpen C, Gorlia T, Mesia $\mathrm{R}$, Degardin M, et al. Cisplatin, fluorouracil, and docetaxel in unresectable head and neck cancer. N.Engl.J.Med. 2007;357(17):1695-704.

27. Paccagnella A, Ghi MG, Loreggian L, Buffoli A, Koussis $\mathrm{H}$, Mione CA, et al. Concomitant chemoradiotherapy versus induction docetaxel, cisplatin and 5 fluorouracil (TPF) followed by concomitant chemoradiotherapy in locally advanced head and neck cancer: a phase II randomized study. Ann.Oncol. 2010;21(7):1515-22.

28. Paccagnella A, Mastromauro C, D'Amanzo P, Ghi MG. Induction chemotherapy before chemoradiotherapy in locally advanced head and neck cancer: the future? Oncologist 2010;15 Suppl 3:8-12.

29. Ghi MG, Paccagnella A, D'Amanzo P, Mione CA, Fasan S, Paro S, et al. Neoadjuvant docetaxel, cisplatin, 5 -fluorouracil before concurrent chemoradiotherapy in locally advanced squamous cell carcinoma of the head and neck versus concomitant chemoradiotherapy: a phase II feasibility study. Int.J.Radiat.Oncol.Biol.Phys. 2004;59(2):481-7.

30. Haddad R, O'Neill A, Rabinowits G, Tishler R, Khuri F, Adkins D, et al. Induction chemotherapy followed by concurrent chemoradiotherapy (sequential chemoradiotherapy) versus concurrent chemoradiotherapy alone in locally advanced head and neck cancer (PARADIGM): a randomised phase 3 trial. Lancet Oncol. 2013;14(3):257-64.

31. Cohen EE, Karrison T, Kocherginsky M, Huang CH, Agulnik M, Mittal P. DeCIDE: A phase III randomized trial of docetaxel (D), cisplatin (P), 5-fluorouracil (F) (TPF) induction chemotherapy (IC) in patients with N2/ N3 locally advanced squamous cell carcinoma of the head and neck (SCCHN). J.Clin.Oncol. 2012;30:550.

32. Le Tourneau C, Jung GM, Borel C, Bronner G, Flesch $\mathrm{H}$, Velten M. Prognostic factors of survival in head and neck cancer patients treated with surgery and postoperative radiation therapy. Acta Otolaryngol 2008;128(6):706-12. 